\title{
TB OR NOT TB? \\ Difficulties in the Diagnosis of Tuberculosis in HIV-negative IMMIGRANTS TO GERMANY
}

\author{
D. D. Singh ${ }^{1,2}$, M. Vogel ${ }^{3}$, I. Müller-Stöver ${ }^{1}$, T. el Scheich 3 , M. Winzer ${ }^{1}$, S. Göbels ${ }^{1}$, F. Hüttig' \\ S. Heinrich ${ }^{3}$, C. MacKenzie ${ }^{4}$, B. Jensen ${ }^{1}$, S. Reuter ${ }^{1}$, D. Häussinger ${ }^{1}$, J. Richter ${ }^{1}$ \\ ${ }^{1}$ University Hospital for Gastroenterology, Hepatology und Infectious Diseases, ${ }^{2}$ University Hospital for Maxillofacial Surgery, \\ ${ }^{3}$ University Hospital for Pediatrics, ${ }^{4}$ Institute for Medical Microbiology und Hospital Hygiene, \\ Faculty of Medicine, Heinrich-Heine-University, Düsseldorf, Germany
}

\begin{abstract}
Background: Immigrants to Germany and their children are at particular risk for tuberculosis (TB).

Methods: 35 Patients (10 male / 25 female aged 2 - 59 years (median 33 years) originating mostly from high incidence countries in Asia (19 [54.3\%]) in Africa (14 $[40.0 \%]$ and East Europe (2 [5.7\%]), attended at the Tropical Medicine Unit were analysed.

Results: Primary clinical presentation was most frequently lymphadenitis (13 [37.1\%]). Other organs involved included bones $(7[20.0 \%])$, central nervous system $(5[14.3 \%])$, urogenital organs $(3[8.6 \%])$, lung (3 [8.6\%]), mediastinum, $(2[5.7 \%])$ and abdomen $(2$ $[5.7 \%])$. ESR was abnormal in $21 / 28(75.0 \%)$, CRP in $20 / 35(57.1 \%)$, and protein electrophoresis in $22 / 26$ $(84.6 \%)$ cases. The tuberculin skin test was strongly positive in all 15 cases where the test had been performed. Tuberculosis interferon gamma release assay (TB-IGRA) was positive in all 35 cases (100\%). PCR for nucleic acids of Mycobacterium (M.) tuberculosis complex was positive in only $7 / 20(35.0 \%)$ cases. $M$. tuberculosis was identified in 32/35 (91.4\%), M. bovis in $2(5.7 \%)$ cases. 1 case was diagnosed clinically. All patients were negative for HIV. Typical histopathology was seen in the 29 cases, where biopsies had been taken. Chest-X-ray did not reveal specific pulmonary lesions in the majority of cases (22/35 [62.9\%]). Diagnosis of TB was mostly delayed (4 to 299 weeks, [median 8]). The most frequent primary suspicion was a malignancy $(17 / 35[48.6 \%])$ while TB was initially suspected in 5 cases only. Diagnosis of TB is impeded by its multifaceted presentation especially in immigrants.
\end{abstract}

Key words: Tuberculosis, Mycobacterium, M. tuberculosis, M. bovis, migrants, children, lymphoma, neoplasia, diagnostics, tuberculin skin test, TBC-IGRA

\section{INTRODUCTION}

Tuberculosis is among the oldest, most widespread and serious of all human infectious diseases [1-3]. Worldwide, owing to population growth, poverty, inequity, suboptimal health services, insufficient distribution of antituberculous drugs and the impact of the AIDS pandemic, there are more cases of tuberculosis today than at any previous time in human history [2].
2 billion people have been estimated by the World Health Organization to be infected by tubercle bacilli [3].

In Germany, immigrants and children of immigrants from high incidence countries constitute a particular risk group [4].

\section{MATERIALS AND Methods \\ Study Location and Patients}

Foreign patients and immigrants especially from tropical countries with unclear diseases or suspected tuberculosis are seen at the Tropical Medicine Unit of the University Hospital for Gatroenterology, Hepatology and Infectious Diseases. Physicians of this Unit perform consultancies in other Departments of the University Hospital, as well as in other hospitals in the region. In the Unit, care is taken for TB patients usually after these have been assessed before in other wards. In other patients coming from tropical countries, suspicion of TB may arise during the diagnostic work up for a suspected tropical disease.

35 Patients (10 male / 25 female aged 2 to 59 years (median 33 years) were analysed in this retrospective study. Most patients originated from high incidence countries in Asia: India (8), Sri Lanka (3), Kazakhstan (2), Viet Nam (2), Myanmar (1), Turkey (1); and Africa: Morocco (5), Ghana (2), Gambia (1), Senegal (1), Cameroon (2), Kenya (1), Congo (1), Angola (1). Two patients originated from Kosovo, another two patients originated from Japan.

\section{Materials And Methods}

Materials and methods comprise the complete diagnostic work-up at the disposal of a University clinic in an industrialised country. Diagnostic methods for tuberculosis included Mendel-Mantoux tuberculin skin test (TST) with intradermal injection of $0.1 \mathrm{~mL}$ of tuberculin PPD RT 23 (Statens Serum Institut, Copenhagen, Denmark), result read after 48 and 72 hours, tuberculosis (TB)-Interferon-gamma-releaseAssay (IGRA) (T-spot ${ }^{\circledR}$, Oxford Immunotec, Marlborough, U.S.A.), Ziehl-Neelsen staining and culture of mycobacteria including TB drug resistance testing as well as PCR for nucleic acids of Mycobacterium (M.) tuberculosis complex. Lymphocellular immune-compe- 
tence was tested by CD4 and CD8 lymphocyte subtype count. HIV-testing was performed in all patients.

\section{RESULTS}

28 of the 35 patients were referred from other University Departments: seven children and adolescents from Paediatrics, six patients from Infectious Diseases, five from Neurosurgery, three from Otorhinolaryngology, two from Maxillofacial Surgery, two from Orthopaedics, one from Haematology, and one from Gynaecology and Obstetrics. Three patients were referred by other hospitals around Düsseldorf. Two patients presented to our service on their own initiative. One patient was referred by his physician, one by his general practitioner, and one patient was the mother of a TB patient.

The most frequent clinical suspicion for the underlying cause of the patients' symptoms was a malignancy $(17 / 35[48.6 \%])$. Only 14/35 (40.0\%) patients were referred because an infectious disease was suspected of which TB was suspected in 5 cases only (among these was the mother of a TB patient referred by the Public Health Office).

\section{HISTORY}

Although some patients were attended in the hospital for acute illness, e.g. for a generalised seizure, usually patients with a more insidious onset of disease presented to the doctor some weeks after the symptoms had begun. (time elapsed between onset of symptoms and first visit to a doctor: 0-12 weeks [median 4 weeks]).

\section{SYMPTOMS}

Main symptoms which prompted the patients to visit a doctor are listed in Table 1.

Table 1. Reported symptoms of tuberculosis patients.

\begin{tabular}{lc}
\hline Lymphnode swelling & $13(37.1 \%)$ \\
Weight loss & $10(28.6 \%)$ \\
Night sweats & $9(25.7 \%)$ \\
Neurological symptoms ${ }^{1}$ & $8(22.9 \%)$ \\
Coughing, respiratory symptoms & $5(14.3 \%)$ \\
Back pain & $3(8.6 \%)$ \\
Fever & $2(5.7 \%)$ \\
Other symptoms $^{2}$ & $9(25.7 \%)$ \\
\hline
\end{tabular}

${ }^{1}$ paralysis (3), seizure (2), visual disturbance (1), deafness (1), headache (1)

2 abscess (3), pathological bone fracture (1), dysphagia (1), perineal pain (1), scrotal swelling (1), haematuria (1), primary Infertility (1)

\section{LABORATORY RESULTS}

ESR was accelerated in $21 / 28(75.0 \%)$, CRP was raised in $20 / 35(57.1 \%)$, protein electrophoresis was abnormal in 22/26 (84.6\%) cases: albumin was decreased in 12 , increased in 2 , alfa-1-globulins were in- creased in 1, alpha-2-globulins were increased in 4 and decreased in 1 case; beta-globulins were increased 1 case, and gamma-globulins were increased in 16 cases, and decreased in 1 case.

\section{TB Diagnostics}

TST was strongly positive in all $15 / 15$ cases (100\%) where the test had been performed: minimum induration was $18 \mathrm{~mm}$ in diameter, the largest $50 \times 70 \mathrm{~mm}$. 11/15 cases developed ulcerations (Fig. 1) which left a hyperpigmented macula for months after the test had been applied. TB-Interferon-gamma-release-Assay (IGRA) was positive in all 35 cases $(100 \%)$. PCR for nucleic acids of $M$. tuberculosis complex was positive in $9 / 24(37.5 \%)$ cases where this method was applied: PCR of secretory samples (bronchoalveolar lavage and gastric juice) was performed in 9 cases. 4 of these case proved to be exclusively extrapulmonary also by imaging and culture. In the remaining 5 cases with proven pulmonary involvement sensitivity of PCR for $M$. $t u$ berculosis complex in respiratory secretions was $60 \%$ (3/5 cases: $1 / 3$ [33.3\%] bronchoalveolar lavage and $2 / 2[100.0 \%]$ gastric juice. Thus, after exclusion of negative PCR results in respiratory excretions of the 4 exclusively extrapulmonary cases the sensitivity of PCR resulted $45.0 \%(9 / 20)$. PCR is not licenced for non-respiratory secretions. However, sensitivity of PCR was comparable in examining non-respiratoy material (46.2\% [6/13]: It was particularly helpful for detecting lymph node TB (5/10 [50\%]) and tuberculous endometritis from menstrual blood (1/1). PCR failed to reveal Mycobacterium nucleic acids in urine in one case of urogenital TB and in one case of osseous TB.

Cultures revealed $M$. tuberculosis in 32/35 (91.4\%) and $M$. bovis in $2(5.7 \%)$ of cases as the causative agent. The latter was found in two Moroccan patients. In one 19 year old Angolan female patient with granulomatous uveitis, mycobacteria were neither detected by PCR nor in culture. However, the prompt response to tuberculostatic therapy confirmed the suspicion of tuberculosis in this case.

CD4 and CD8 lymphocyte counts and ratios were normal in the $6 / 6$ patients where this test was performed. HIV-testing was negative in all 35 patients.

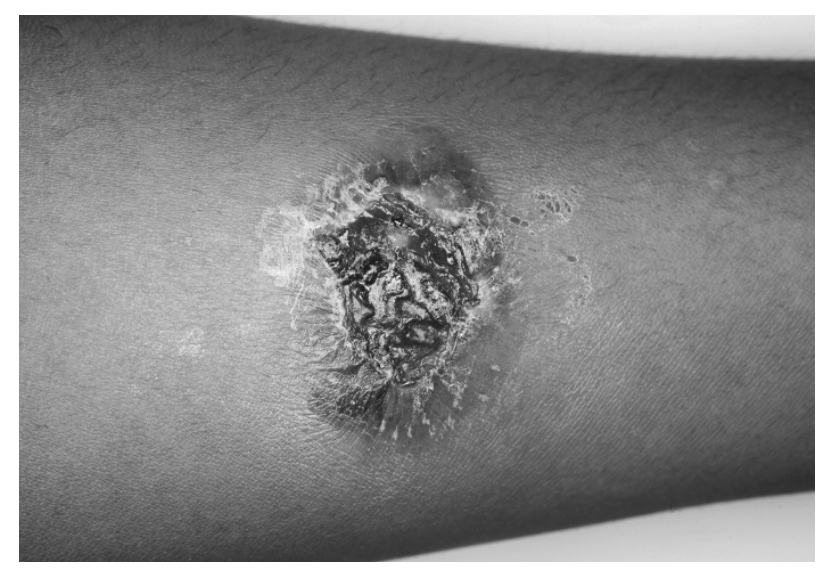

Fig 1. Strongly positive, ulcerative skin reaction to tuberculin in an Indian TB patient. 
Chest-X-ray showed suspicious lesions in 13/35 $(37.1 \%)$ cases only. Histopathology findings with typical caseous granulomas infiltrated by giant cells of Langerhans type were seen in 29 cases where guided biopsies had been performed.

\section{Clinical Classification of Patients}

Primary clinical presentation was most frequently lymphadenitis, followed by osseous involvement, involvement of the central nervous system, urogenital organs, lung, mediastinum and abdomen (Table 2). Patients were classified into exclusively extrapulmonary, exclusively pulmonary and pulmonary+extrapulmonary dissemination. In $4 / 36(11.1 \%)$ patients acid fast bacilli were seen in smear or bronchoalveolar lavage (open pulmonary tuberculosis). In sputum, bronchoalveolar lavage and/or gastric juice of further 9 patients tu-

Table 2. Primary clinical presentation of tuberculosis cases.

\begin{tabular}{ll}
\hline Lymph nodal & $13(37.1 \%)$ \\
$\quad$ Cervical & $12(2$ submandibular $)$ \\
$\quad$ Axillary & 1 \\
Osseous & $7(20.0 \%)$ \\
Central nervous & $5(14.3 \%)$ \\
Urogenital & $3(8.6 \%)$ \\
Pulmonary & $3(8.6 \%)$ \\
Medianistinal & $2(5.7 \%)$ \\
Abdominal & $2(5.7 \%)$ \\
\hline
\end{tabular}

Table 3. Classification of tuberculosis cases.

\begin{tabular}{ll}
\hline Extrapulmonary TB & $\mathbf{2 2}(\mathbf{6 2 . 9 \% )}$ \\
$\quad$ Lymphadenitis & $10(28.6 \%)$ \\
Osseous & $6(17.1 \%)$ \\
Urogenital & $2(5.7 \%)$ \\
CNS & $2(5.7 \%)$ \\
Mediastinal & $1(2.9 \%)$ \\
Pleural & $1(2.9 \%)$ \\
& \\
Pulmonary TB* & $\mathbf{1 3}(\mathbf{3 7 . 1 \% )}$ \\
Disseminated & $10 / 13(76.9 \%)$ \\
Lymphadenitis+pulmonary & 1 \\
Lymphadenitis+abdominal+pulmonary & 1 \\
Lymphadenitis+mediastinal+pulmonary & 1 \\
Osseous+lymphadenitis+pleural-pulmonary & 1 \\
CNS+pulmonary & 1 \\
CNS+lymphadenitis+pulmonary & 1 \\
CNS+osseous+pulmonary & 1 \\
Abdominal+pleural+thymus+pulmonary & 1 \\
Abdominal+pulmonary & 1 \\
Scrotal+pulmonary & 1 \\
Restricted to the lungs & $3 / 13(23.1 \%)$ \\
\hline
\end{tabular}

*Smear-positive: 4/13 (30.8\%); smear negative - culture-positive: $9 / 13(69.2 \%)$
Table 4. Primary clinical suspicion of tuberculosis cases.

\begin{tabular}{lr}
\hline Malignancies & \\
$\quad$ Lymphoma & 10 \\
$\quad$ Other malignancies & 7 \\
Tuberculosis & 5 \\
Osteomyelitis & 3 \\
Abscess & 2 \\
Sarcoidosis & 2 \\
Hormonal dysfunction & 1 \\
Otitis & 1 \\
Schistosomiasis & 1 \\
Tropical infection & 1 \\
AIDS & 1 \\
Multiorganic systemic disease & 1
\end{tabular}

berculosis bacteria could be detected by culture alone. The majority of cases (22/35 [62.9\%]) had no pulmonary primary complex. Only $3 / 35$ [8.6\%] of cases had involvement restricted to the lung. Most of the other cases with pulmonary involvement had additional disseminated lesions in other organs. (Table 3 )

TB was rarely initially suspected by the clinicians (Table 4). First suspicion of the clinicians included malignancies, autoimmune diseases and other infections. The diagnosis was delayed: the time between the first visit to a doctor and established TB diagnosis lasted from 4 to 299 weeks (median 8 weeks).

\section{DisCusSION}

The delayed diagnosis of tuberculosis in our patients may be due to the multifaceted clinical presentation of the disease and to the decreasing familiarity of physicians with this infection in Germany. TB in native patients of German origin most frequently present as pulmonary TB in elderly, debilitated patients [4]. This is one of the reasons why the TB control in Germany after World War II based on mass chest-X-ray screening was successful. Moreover, the delayed diagnosis may be partly due to the particular characteristics of the selected patient collective observed, i.e. HIV-negative immigrants which is not representative for all TB patients notified. For instance, in 2009 in Düsseldorf 60 new cases (31 male / 29 female; 32\% German, 68\% immigrants or foreigners) have been notified. Among these, $72 \%$ had pulmonary involvement, $28 \%$ were exclusively extrapulmonary. In our cohort, most patients were female and young and had neither respiratory complaints nor B symptoms. In tropical regions, exclusively extrapulmonary presentation of TB is relatively frequent, a notion confirmed by our cohort. [2, 4-5]. Furthermore, TB is usually deemed to increase inflammatory parameters such as ESR, or CRP. These are not only non-specific, but surprisingly, a number of patients even with disseminated TB had normal ESR and/or CRP. The most sensitive parameter would have been an abnormal protein electrophoresis. Nowadays TB-IGRA's are preferred to TST because the IGRA's are more sensitive and more specific than TST [6-11]. Especially, TB-IGRA's although also covering $M$. bovis 
are not confounded by previous BCG vaccination [7]. In particular, for the patient collective described, we do not share the opinion that TST is obsolete: firstly, at least in adult immigrants from countries with high TB incidence a positive TB-IGRA does not enable to differentiate between latent inactive and active TB. If we assume that the more than 2 billion TB cases worldwide most frequently occur in high incidence countries, a positive IGRA may be expected in more than one third of people from high incidence countries at least from adolescence on [3]. Secondly, although the TST is usually less sensitive and less specific than TB-IGRA, in our cohort false negative results did not occur. Thirdly, and most importantly, contrary to TB-IGRA, skin reaction to tuberculin can be semi-quantified into weakly to strongly positive. So far, for TB-IGRA, there is no quantitative assessment which would prove active TB [12]. On the other hand, in our patients without overt immuno-incompetence suspicion of TB mostly aroused by the intensity of their skin reaction to tuberculin. This is why, for the moment, we see both tests as complementary tools rather than as mutually exclusive. In future, TB-IGRA may be useful for the diagnosis of active TB when applied to body materials other than blood, e.g. bonchoalveolar lavage, cerebrospinal fluid, pericardial, pleural or peritoneal effusions [13-17]. Performance of PCR for nucleic acids of $M$. tuberculosis was unsatisfactory. Sensitivity of PCR as compared to culture was lower than $50 \%$. PCR did not perform better in its licensed application on respiratory materials than in its application on other materials. However, a positive PCR was very helpful for speeding up the diagnostic progress and initiation of therapy because of the rather long time interval a TB culture takes to become positive.

\section{CONCLUSION}

Tuberculosis in immigrants has particular multifaceted presentations which have to be taken into account by the physician. In immigrants diagnosis is difficult because pulmonary involvement is not constant.

Acknowledgement: This work is part of a medical thesis by Daman Deep Singh. We are indepted to Dr. Ursula Lang, Public Health Office Düsseldorf for communicating us the TB statistic of the Office.

\section{REFERENCES}

1. Donahugue HD, Spigelman M, Greenblatt CL, LevMaor G, Bar-Gal GK, Mathe-son C, Vernon K, Nerlich AG, Zink AR Tuberculosis: from prehistory to Robert Koch, as revealed by ancient DNA. Lancet Infect Dis. 2004; 4: 584-92.

2 Grange JM \& Zumla AI. Tuberculosis 2009 in: GC Cook \& AI Zumla eds.: Man-son's Tropical Diseases 22nd ed. Saunders Philadelphia, U.S.A. pp. 983-1051.

3. World Health Organization. Global Tuberculosis Control: Surveillance, Planning, Financing. WHO Report 2009; World Health Organization, Geneva; www.who.int $>$ WHO sites $>$ Health Topics $>$ Tuberculosis $>$ Publications

4. Infektionsepidemiologisches Jahrbuch meldepflichtiger Krankheiten für 2009. Ro-bert Koch Institut, Berlin, Germany: 2010: pp: 182-186.
5. Rüsch-Gerdes S \& Hillemann D. Tuberkulose und nicht tuberkulöse Mykobakterio-sen. In T Löscher, GD Burchard eds.: Tropenmedizin in Klinik und Praxis. 4th ed. Thieme Stuttgart, Germany 2010: pp. 444-458.

6. Huebner R E, Schein M F, Bass J B Jr. The tuberculin skin test. Clin Infect Dis. 1993; 17: 968-975.

7. Wang L, Turner MO, Elwood RK, Schulzer M, FitzGerald JM. A meta-analysis of the effect of Bacille Calmette Guerin vaccination on tuberculin skin test measurements. Thorax. 2002 Sep;57(9):804-809.

8. Delgado J C, Tsai E Y, Thim S, et al. Antigen-specific and persistent tuberculin anergy in a cohort of pulmonary tuberculosis patients from rural Cambodia. Proc Natl Acad Sci USA. 2002; 99: 7576-7581.

9. Horsburgh C R Jr. Priorities for the treatment of latent tuberculosis infection in the United States. N Engl J Med. 2004; 350: 2060-2067.

10. Diel R, Loddenkemper R, Niemann S, Meywald-Walter K, Nienhaus A. Negative and positive predictive value of a whole-blood interferon-gamma release assay for developing active tuberculosis. An Update. Am J Resp Crit Care Med. 2011, 183; 88-95.

11. Bamford AR, Crook AM, Clark JE, Nademi Z, Dixon G, Paton JY, Riddel A, Drob-niewski F, Riordan A, Anderson S, Williams A, Walters S, Kampmann B. Comparison of interferon-gamma release assays and tuberculin skin test in predicting active tuber-culosis (TB) in children in the UK: a paediatric TB network study. Arch Dis Child. Mar 2010; 95(3):180-6.

12. Kampmann B, Wittaker E, Williams A., Walters S, Gordon A, Martinez-Alier N, Williams B, Crook AM. Interferon-gamma release assays do not identify more children with active tuberculosis than the tuberculin skin test. Eur Resp J. 2009; 33:1371-1379.

13. Lorenz R, Wurl P, Haerter G, Cammerer G, Barth T, Hausladen S, Stenger S, Kern W. Interferon-gamma release assay in the ascites: Early hint for diagnosis of abdominal tuberculosis. Infection 2010. Feb;38(1):69-72.

14. Biglino A, Crivelli P, Concialdi E, Bolla C, Montrucchio G. Clinical usefulness of ELISPOT assay on pericardial fluid in a case of suspected tuberculous pericarditis. Infection. 2008 Dec;36(6):601-4.

15. Kosters K, Nau R, Bossink A, Greiffendorf I, Jentsch M, Ernst M, et al. Rapid di-agnosis of CNS tuberculosis by a T-cell interferon-gamma release assay on cerebro-spinal fluid mononuclear cells. Infection. 2008 Dec;36(6):597-600.

16. Jafari C, Ernst M, Kalsdorf B, Greinert U, Diel R, Kirsten $\mathrm{D}$, et al. Rapid diagnosis of smear-negative tuberculosis by bronchoalveolar lavage enzyme-linked immuno-spot. Am J Respir Crit Care Med. 2006 Nov 1;174(9):1048-54.

17. Lange C, Pai M, Drobniewski F, Migliori GB. InterferonGamma release assays fort he diagnosis of active tuberculosis: sensible or silly?. Eur Resp J. 2009; 33:1250-1253.

18. Schreiber J, Meyer C, Rüsch-Gerdes S, Richter E, Beck $\mathrm{H}$, Fischer JF, Rosahl W. Mycobacterium tuberculosis gene-amplification in breath condensate of patients with lung tuberculosis. Eur J Med Res. 2002 Jun 28;7(6):290-1.

Received: April 20, 2011 / Accepted: May 26, 2011

Address for correspondence:

Prof. Dott. Univ. Pisa Joachim Richter

Tropenmedizinische Ambulanz; Geb. 14.78

Klinik für Gastroenterologie, Hepatologie und Infektiologie

Universitätsklinikum Düsseldorf

Moorenstr. 5

D-40225 Düsseldorf

Germany

Tel.: +49-211-8116800; +49-211-8119161

Fax: +49-211-3190639

E-mail: Joachim.Richter@med.uni-duesseldorf.de 